\title{
8-ary modulation with triangular lattice
}

\author{
Sung-Joon Park ${ }^{1,}$ a) \\ ${ }^{1}$ Department of Electronic Engineering, Gangneung-Wonju National Universtiy \\ 7 Jukheon-gil, Gangneung, Gangwon 25457, Republic of Korea \\ a)psj@ieee.org
}

Abstract: High-order modulation is currently widespread since it increases spectral efficiency and data rate. In case of 8-ary signal set, the 8-ary phase shift keying having a constant envelope is useful but presents a poor performance in terms of power efficiency. In this letter, 8-ary modulation with a triangular lattice is investigated to improve the performance of 8-ary signaling and its power gains are elaborated analytically. According to results, the proposed 8-ary signal set provides the asymptotic power gains of $1.811 \mathrm{~dB}$ and $0.218 \mathrm{~dB}$ over the conventional 8-ary phase shift keying and quadrature amplitude modulation, respectively.

Keywords: phase shift keying, quadrature amplitude modulation, triangular lattice, power gain

Classification: Transmission Systems and Transmission Equipment for Communications

\section{References}

[1] C.R. Cahn, "Combined digital phase and amplitude modulation communication systems," IRE Trans. Commun., vol. 8, no. 3, pp. 150-155, Sept. 1960. DOI: 10.1109/TCOM.1960.1097623

[2] J.C. Hancock and R.W. Lucky, "Performance of combined amplitude and phase-modulated communication systems," IRE Trans. Commun., vol. 8, no. 4, pp. 232-237, Dec. 1960. DOI: 10.1109/TCOM.1960.1097638

[3] G. Foschini, R. Gitlin, and S. Weinstein, "Optimization of two-dimensional signal constellations in the presence of Gaussian noise," IEEE Trans. Commun., vol. 22, no. 1, pp. 28-38, Jan. 1974. DOI: 10.1109/TCOM.1974.1092061

[4] L. Hanzo, W. Webb, and T. Keller, Single and Multi-Carrier Quadrature Amplitude Modulation, John Wiley \& Sons, 2000.

[5] C. Campopiano and B. Glazer, "A coherent digital amplitude and phase modulation scheme," IRE Trans. Commun., vol. 10, no. 1, pp. 90-95, March 1962. DOI: 10.1109/TCOM.1962.1088634

[6] S.-J. Park, "Triangular quadrature amplitude modulation," IEEE Commun. Lett., vol. 11, no. 4, pp. 292-294, April 2007. DOI: 10.1109/LCOM.2007.348278

[7] S.-J. Park, "Irregular triangular quadrature amplitude modulations," IEICE Trans. Commun., vol. E97-B, no. 7, pp. 1358-1364, July 2014. DOI: 10.1587/ transcom.E97.B.1358

[8] J.G. Proakis and M. Salehi, Digital Communications, Mc-Graw Hill, 2008.

[9] J.G. Smith, "Odd-bit quadrature amplitude shift keying," IEEE Trans. Commun., vol. 23, no. 3, pp. 385-389, March 1975. DOI: 10.1109/TCOM.1975.1092806 
[10] E.N. Gilbert, "A comparison of signalling alphabets," Bell Syst. Tech. J., vol. 31, no. 3, pp. 504-522, May 1952. DOI: 10.1002/j.1538-7305.1952.tb01393.x

\section{Introduction}

Since a high-order modulation enables high-speed data transfer, signal constellations possessing good properties have been searched for decades [1, 2, 3, 4]. Among them, square quadrature amplitude modulation (SQAM) which takes the constellation of a square lattice is the most prevalent high-order modulation technique owing to reasonable performance and simple detection [5].

Recently, triangular quadrature amplitude modulation (TQAM) has been proposed for the $M$-ary signal sets, $M=2^{2 m}, m=2,3, \cdots$, where signal points are located at the vertexes of a triangular lattice [6]. It ensures the improved power efficiency at the expense of slight increase in complexity for demodulation when compared with the SQAM. Further, it is proven that a more power gain could be achieved by relaxing the constraint of regularity on the placement of signal points [7]. From the observation that the well-known 8-ary phase shift keying (PSK) is not efficient in terms of the use of symbol energy, in this letter, a TQAM for 8-ary signal set is proposed and analysed. Also, the performance of the 8-ary TQAM is compared with the performance of the 8-ary PSK and QAM.

The remainder of this letter is organized as follows. First, the signal constellation of the 8-ary TQAM is addressed with other notable constellations. Then, the error rates, power gains, and peak-to-average power ratio (PAPR) of the proposed scheme are elaborated and analysed. Finally, a brief summary is provided.

\section{Signal constellation}

Figure 1 shows the 8-ary PSK, the 8-ary QAM denoted as the best constellation with two amplitudes and a constant minimum distance [8, Ch.4.3.3], and the proposed 8 -ary TQAM having signal points located at the vertexes of a triangular lattice, respectively, where $d$ denotes a half of minimum distance. If the rule generating the irregular TQAM suggested in [7] is applied to 8-ary signal set, the first signal point of the 8-ary TQAM should be placed at $(d, 0)$. Then, the other points are located one by one at the vertexes of a triangular lattice while minimizing the average energy

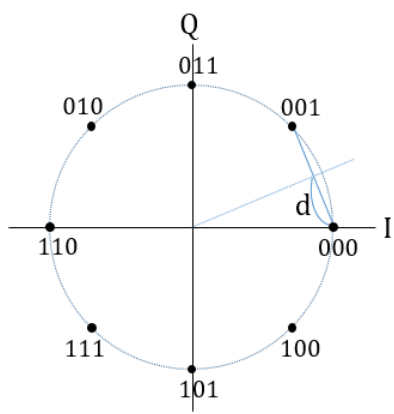

(a) 8-ary PSK

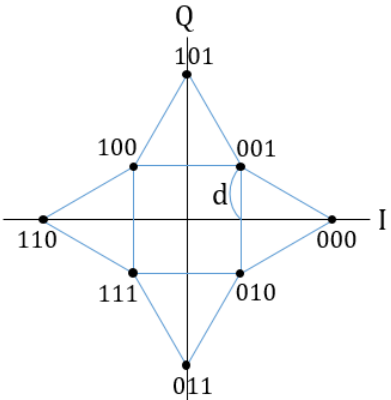

(b) 8-ary QAM

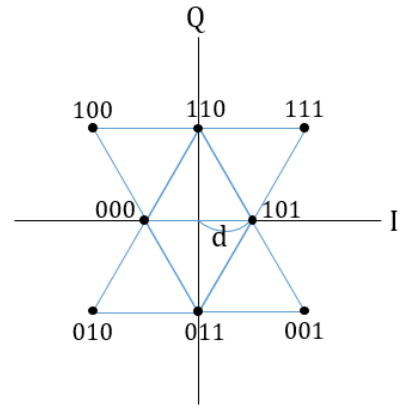

(c) 8-ary TQAM

Fig. 1. Signal constellations for 8-ary signal set. 
per symbol $E_{s}$. The resultant signal constellation is shown in Fig. 1(c) and it is symmetric with respect to both in-phase and quadrature axes. Further, the bit stream mappings for the 8-ary QAM and TQAM are obtained by a full search with the cost function minimizing the Gray coding penalty $G_{p}$, which is defined as the average number of bit differences between a symbol and its nearest neighbors [9].

\section{Performance analysis}

The well-known approximation on symbol error rate (SER), $P_{S}$ and bit error rate (BER) $P_{b}$ suggested in [10] is given by

$$
\begin{gathered}
P_{s}=N Q\left(\sqrt{2 / N_{0}} d\right) \\
P_{b}=\frac{G_{p}}{\log _{2} M} P_{S}
\end{gathered}
$$

where $N$ represents the average number of the nearest neighbors of each signal point, $N_{0} / 2$ means the two-sided noise power spectral density, and $Q(\cdot)$ denotes the Gaussian Q-function. The parameters in (1) and (2) for the 8-ary TQAM are calculated from the constellation shown in Fig. $1 c$ and compared with those of the 8-ary PSK and 8-ary QAM in Table I.

Table I. Parameters for performance analysis

\begin{tabular}{l|l|l|l}
\hline & 8 -ary PSK & 8 -ary QAM & 8-ary TQAM \\
\hline$E_{S} / d^{2}$ & 6.828 & 4.732 & 4.5 \\
\hline$N$ & 2 & 3 & 3.25 \\
\hline$G_{p}$ & 1 & 1.25 & 1.275 \\
\hline
\end{tabular}

From (1) and Table I, the SERs of the 8-ary TQAM and PSK are obtained by

$$
\begin{gathered}
P_{S, T Q A M}=3.25 Q\left(\frac{2}{3} \sqrt{\left(\frac{E_{S}}{N_{0}}\right)_{T Q A M}}\right) \\
P_{S, P S K}=2 Q\left(\sqrt{2} \sin \left(\frac{\pi}{8}\right) \sqrt{\left(\frac{E_{S}}{N_{0}}\right)_{P S K}}\right) .
\end{gathered}
$$

Thus, for the same target SER, the following equation is approximately hold if we ignore the coefficient on the right-hand side in (3) and (4).

$$
\frac{2}{3} \sqrt{\left(\frac{E_{S}}{N_{0}}\right)_{T Q A M}}=\sqrt{2} \sin \left(\frac{\pi}{8}\right) \sqrt{\left(\frac{E_{S}}{N_{0}}\right)_{P S K}}
$$

By elaborating on (5), the asymptotic power gain of the 8-ary TQAM over the 8-ary PSK is obtained in linear and dB scales, respectively.

$$
\begin{gathered}
\frac{\left(\frac{E_{S}}{N_{0}}\right)_{P S K}}{\left(\frac{E_{s}}{N_{0}}\right)_{T Q A M}}=\frac{2}{9 \sin ^{2}(\pi / 8)}=1.517 \\
10 \log \left(\frac{E_{S}}{N_{0}}\right)_{P S K}-10 \log \left(\frac{E_{S}}{N_{0}}\right)_{T Q A M}=1.811 \mathrm{~dB}
\end{gathered}
$$

In a similar way, the asymptotic gain of the 8-ary TQAM over the 8-ary QAM is calculated as $0.218 \mathrm{~dB}$. 
Figure 2 is a plot of the power gains of the 8-ary TQAM over PSK and QAM as a function of a target error rate. In the figure, the actual gains for an error rate are numerically acquired from (1), (2) and Table I, and the asymptotic power gains are included for comparison. From the results, it is observed that the actual gains converge to the ideal values with the increase of error rate and are quite large even at a low probability of error. For example, at a target error rate of $10^{-6}$, the gains of TQAM over PSK amount to $1.645 \mathrm{~dB}$ in SER and $1.541 \mathrm{~dB}$ in BER and the gains of TQAM over QAM amount to $0.191 \mathrm{~dB}$ in SER and $0.182 \mathrm{~dB}$ in BER. Further, it is found that the BER gain is smaller than the SER gain since the Gray coding penalty $G_{p}$ of TQAM is larger than those of PSK and QAM in (2).

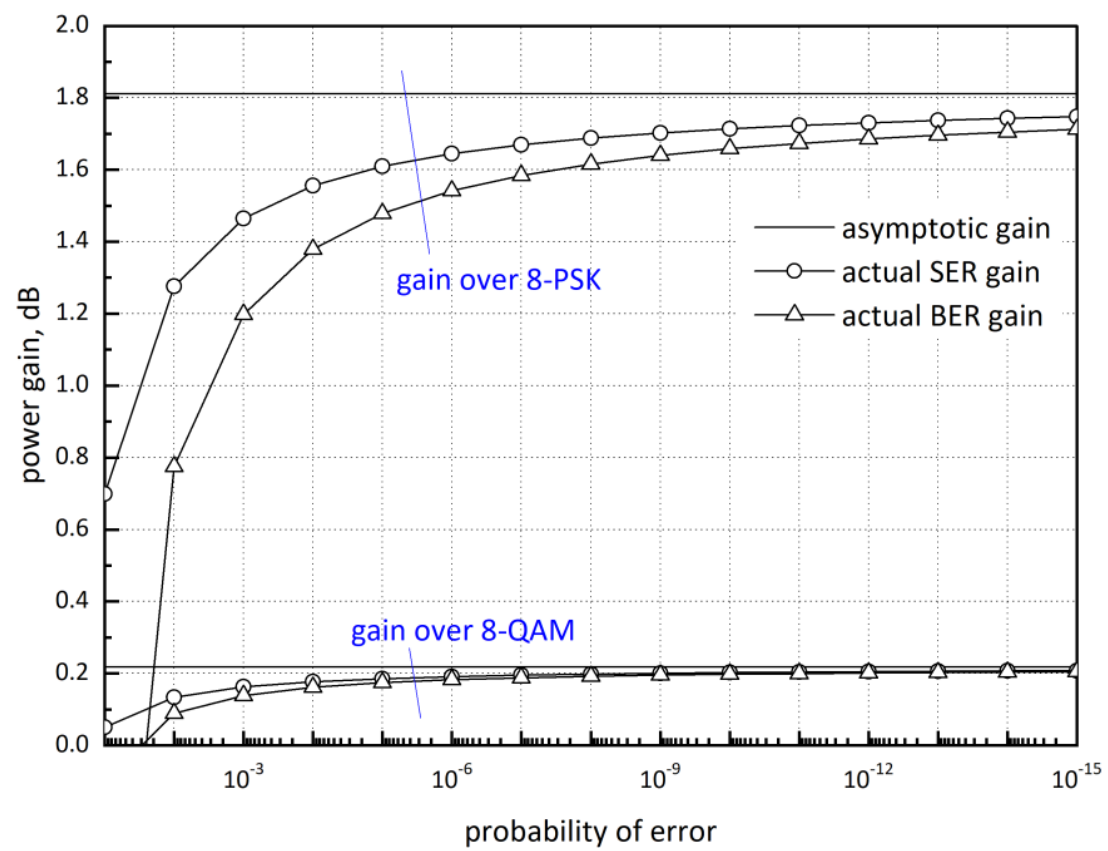

Fig. 2. Power gains with respect to probability of error.

The main disadvantage of the 8-ary TQAM is that it does not maintain a constant envelope, causing an increase in the PAPR. From the observation that the 8-ary TQAM has the signal points located at one of the three concentric circles having the radius $d, \sqrt{3} d$ or $\sqrt{7} d$, its PAPR is easily calculated as

$$
\operatorname{PAPR}_{T Q A M}=\frac{\text { Peak power }}{\text { Average power }}=\frac{7 d^{2}}{\frac{9}{2} d^{2}}=1.556 .
$$

As expected, this value is larger than one, i.e., the PAPR of the 8-ary PSK. Interestingly, according to calculation, the PAPR of 8-ary QAM becomes 1.577 which is larger than the PAPR of 8-ary TQAM. Thus, the 8-ary TQAM has an advantage over the 8-ary QAM in both power efficiency and PAPR.

\section{Conclusion}

The signal constellation of 8-ary signal set forming a symmetrical triangular lattice has been proposed and the optimum bit stream mapping has been found by a full search. From the analysis including the derivation of error rates of the 8-ary TQAM, 
it has been revealed that the asymptotic power gains of the 8-ary TQAM over PSK and QAM amount to $1.811 \mathrm{~dB}$ and $0.218 \mathrm{~dB}$. Further, according to the numerical results, a significant power gain could be achieved even at a low target error rate, making the proposed constellation useful.

\section{Acknowledgments}

This work was supported in part by NRF grant funded by the MSIT (NRF2020R1F1A1048594), and in part by the research grant of Gangneung-Wonju National University. 